\section{Brief Communication}

\section{Check for updates}

\section{OPEN ACCESS}

Received: Sep 11, 2019

Revised: Oct 28, 2019

Accepted: Nov 8, 2019

Correspondence to

Kyung Won Kim, MD, PhD

Department of Pediatrics, Severance Hospital, Institute of Allergy, Brain Korea 21 PLUS Project for Medical Science, Yonsei University College of Medicine, 50-1 Yonsei-ro, Seodaemun-gu,

Seoul 03722, Korea.

Tel: +82-2-2228-2050

E-mail: kwkim@yuhs.ac

\section{Jae Myun Lee, MD, PhD}

Department of Microbiology and Immunology, Brain Korea 21 PLUS Project for Medical

Science, Yonsei University College of Medicine, 50-1 Yonsei-ro, Seodaemun-gu, Seoul 03722, Korea.

Tel: +82-2-2228-1835

E-mail: jaemyun@yuhs.ac

Copyright $(2020$ The Korean Academy of Asthma, Allergy and Clinical Immunology . The Korean Academy of Pediatric Allergy and Respiratory Disease

This is an Open Access article distributed under the terms of the Creative Commons Attribution Non-Commercial License (https:// creativecommons.org/licenses/by-nc/4.0/) which permits unrestricted non-commercial use, distribution, and reproduction in any medium, provided the original work is properly cited.

ORCID iDs

Min Jung Kim (ID

https://orcid.org/0000-0002-5634-9709 Doo Hee Shim (D)

https://orcid.org/0000-0002-6696-7199

\title{
Delayed-Onset Anaphylaxis Caused by IgE Response to Influenza Vaccination
}

\author{
Min Jung Kim $\left(\mathbb{D},{ }^{1,2}\right.$ Doo Hee Shim $\mathbb{D}^{1},{ }^{2}$ Hye-Ran Cha $\mathbb{D},{ }^{2}$ Cheong Bi Kim $\mathbb{D},{ }^{2}$ \\ Soo Yeon Kim (i), Jeon Han Park (1), ${ }^{2}$ Myung Hyun Sohn $\left(\mathbb{D},{ }^{1}\right.$ Jae Myun Lee $\left(\mathbb{D},,^{2^{*}}\right.$ \\ Kyung Won Kim (ib ${ }^{*}$
}

'Department of Pediatrics, Severance Hospital, Institute of Allergy, Institute for Immunology and Immunological Diseases, Brain Korea 21 PLUS Project for Medical Science, Yonsei University College of Medicine, Seoul, Korea

${ }^{2}$ Department of Microbiology and Immunology, Institute for Immunology and Immunological Diseases, Brain Korea 21 PLUS Project for Medical Science, Yonsei University College of Medicine, Seoul, Korea

\section{ABSTRACT}

Influenza vaccine-associated anaphylaxis is a very rare allergic reaction to vaccines, but the most concerning and life-threatening adverse reaction. Although the safety of influenza vaccines has been well documented, occasional cases of anaphylaxis in vaccinated patients have been reported. In this study, we analyzed the immunoglobulin E (IgE) response to whole influenza vaccines in a pediatric case of delayed-onset anaphylaxis after influenza vaccination. The patient showed elevated specific IgE levels against whole influenza vaccines, especially with split virion from egg-based manufacturing process. Specific IgE levels to influenza vaccines showed decreased over. We evaluated a causal relationship between influenza vaccine and anaphylaxis event by enzyme-linked immunosorbent assay. Delayedonset anaphylaxis after influenza vaccination can occur in children without predisposing allergic diseases. In addition, the results suggested that formulation and production system of influenza vaccines could affect the probability of severe allergic reaction to vaccines.

Keywords: Anaphylaxis; drug hypersensitivity; immunoglobulin E; influenza; vaccination; vaccines; delayed hypersensitivity

\section{INTRODUCTION}

Vaccine-associated anaphylaxis is extremely rare, but the most concerning and lifethreatening allergic reaction to vaccines. ${ }^{1,2} \mathrm{~A}$ typical vaccine-associated anaphylaxis has been known to occur within $30 \mathrm{~min}$ after immunization, but delayed-onset anaphylaxis could occur at $>4$ hours after immunization. ${ }^{1}$ A reaction occurring at $>4$ hours after immunization but affecting 2 or more systems could be defined as anaphylaxis of "possible anaphylactic reaction." ${ }^{3}$ However, it is sometimes hard to differentiate anaphylaxis from other adverse reactions and to prove the association between anaphylaxis and vaccines. Therefore, identification of the causal relationship is critical for the diagnosis, management, and prophylaxis in vaccine-associated anaphylaxis. ${ }^{1,2,4}$

Influenza vaccination is annually recommended for all persons older than 6 months, because formulation of vaccines could be changed by season. ${ }^{1,2}$ To influenza vaccines, anaphylaxis occurs at a rate of 1.35 cases per million doses. ${ }^{4,5}$ Most of these cases included acute-onset 
Hye-Ran Cha iD

https://orcid.org/0000-0001-7112-7525

Cheong Bi Kim (iD)

https://orcid.org/0000-0002-9791-4639

Soo Yeon Kim (iD

https://orcid.org/0000-0003-4965-6193

Jeon Han Park (iD)

https://orcid.org/0000-0001-9604-3205

Myung Hyun Sohn iD

https://orcid.org/0000-0002-2478-487X

Jae Myun Lee iD

https://orcid.org/0000-0002-5273-3113

Kyung Won Kim (iD

https://orcid.org/0000-0003-4529-6135

Disclosure

There is no financial or other issue that might

lead to a conflict of interest. symptoms in patients with predisposing allergic diseases. ${ }^{4,5}$ Herein, we present a case of delayed-onset anaphylactic shock caused by immunoglobulin E (IgE) response to a seasonal influenza vaccine in a child without a history of allergic diseases or sensitization.

\section{MATERIALS AND METHODS}

\section{A clinical case}

A 3-year-old-girl was admitted to our Emergency Department, with facial rashes and edema, periorbital and lip swelling, and abdominal pain. Initial vital signs presented as blood pressure, $82 / 54 \mathrm{mmHg}$; pulse rate, 121/min; and respiratory rate 35/min. Generalized skin rashes and itching sensation did not subside after treatment with steroids and antihistamines. Respiratory difficulty and hypotension with tachycardia (blood pressure, $74 / 40 \mathrm{mmHg}$; pulse rate, $175 / \mathrm{min}$; and respiratory rate, $45 / \mathrm{min}$ ) also developed despite adequate intravenous hydration. Under the diagnosis of anaphylactic shock, ${ }^{6}$ the patient was treated with intramuscular injection of epinephrine twice, but blood pressure and pulse rate were remained unstable. After central venous catheter insertion and intravenous epinephrine administration, the patient was transferred to the pediatric intensive care unit for intensive monitoring and treatment. On the subsequent day, vital signs became stable without epinephrine administration, she moved to general ward.

She was vaccinated with a seasonal quadrivalent influenza vaccine the previous day. These symptoms were recognized 12 hours after vaccination, and generalized erythema and itching were aggravated. She had no physician-diagnosed allergic diseases, but only had experienced urticaria after trivalent influenza vaccine administration the previous year. Other laboratory findings, including allergy test, were not significant (total serum IgE level, $23.9 \mathrm{kUA} / \mathrm{L}$; egg white-specific level, 0.04; ovalbumin-specific level, 0.02; and ovomucoid-specific IgE level,0.01 kUA/L, respectively; ImmunoCAP ${ }^{\circledR}$, Thermo Fisher Scientific, Waltham, MS, USA).

\section{Measurement of specific IgE levels to influenza vaccines}

We collected blood samples from the patient on days 1, 3, and 7 after anaphylaxis. The specific IgE levels in response to whole influenza vaccines from different manufacturers were measured by enzyme-linked immunosorbent assay (ELISA). ${ }^{2}$ Briefly, plasma specimens diluted with blocking buffer $(0.2 \%$ skim milk in phosphate-buffered saline containing $0.05 \%$ Tween-20) were incubated with antigens from whole influenza vaccines overnight at $4^{\circ} \mathrm{C}$. Then, horseradish-peroxidase-conjugated goat anti-human IgE (1:5,000; Abcam, Cambridge, United Kingdom) was added and incubated for 1 hour at room temperature. After the reaction, the absorbance was measured at $450 \mathrm{~nm}$.

The investigated inactivated vaccines are listed in Table, produced from 4 different manufacturers in 2016-2017 season. Two trivalent (vaccine A and E) and 2 quadrivalent influenza vaccines (vaccines B and C) were included. Vaccine A was administered the previous year, and present symptoms occurred after the injection of vaccine B. Vaccine D, produced for 2012-2013 season, was tested for comparison with vaccine E. All vaccines contained ovalbumin at a concentration of $\leq 0.1 \mu \mathrm{g} / \mathrm{mL}$. They did not contain adjuvant, gelatin, thimerosal, or 2-phenoxyethanol. We did not consider preservatives or stabilizers in the vaccines.

Data were analyzed using Prism v.6.04 software (GraphPad Inc., La Jolla, CA, USA). This study was approved by the Institutional Review Board of Severance Hospital (Seoul, Korea; 
Table. Types of influenza vaccines

\begin{tabular}{|c|c|c|c|c|c|c|c|}
\hline \multirow[t]{2}{*}{ Vaccine } & \multirow[t]{2}{*}{ Manufacturer } & \multirow{2}{*}{\multicolumn{2}{|c|}{ Trivalent or quadrivalent }} & \multicolumn{2}{|c|}{ Type of propagation } & \multicolumn{2}{|c|}{ Type of antigen } \\
\hline & & & & Embryonated egg-based & Cell culture-based & Split & Subunit \\
\hline$A$ & 1 & Tri & $\begin{array}{l}\text { A/California/7/2009 (H1N1) pdm09-like strain, } \\
\text { A/Hong Kong/4801/2014 (H3N2)-like strain, } \\
\text { B/Brisbane/60/2008-like strain }\end{array}$ & O & & O & \\
\hline B & 1 & Quad & $\begin{array}{l}\text { A/California/7/2009 (H1N1) pdm09-like strain, } \\
\text { A/Hong Kong/4801/2014 (H3N2)-like strain, } \\
\text { B/Brisbane/60/2008-like strain, } \\
\text { B/Puhket/3073/2013-like strain }\end{array}$ & O & & $\bigcirc$ & \\
\hline C & 2 & Quad & $\begin{array}{l}\text { A/California/7/2009 (H1N1) pdm09-like strain, } \\
\text { A/Hong Kong/4801/2014 (H3N2)-like strain, } \\
\text { B/Brisbane/60/2008-like strain, } \\
\text { B/Puhket/3073/2013-like strain }\end{array}$ & O & & $\mathrm{O}$ & \\
\hline D & 3 & Tri & $\begin{array}{l}\text { A/California/7/2009 (H1N1) pdm09-like strain, } \\
\text { A/Perth/16/2009 (H3N2)-like strain, } \\
\text { B/Brisbane/60/2008-like strain }\end{array}$ & O & & & O \\
\hline$E$ & 4 & Tri & $\begin{array}{l}\text { A/California/7/2009 (H1N1) pdm09-like strain, } \\
\text { A/Hong Kong/4801/2014 (H3N2)-like strain, } \\
\text { B/Brisbane/60/2008-like strain }\end{array}$ & & O & & O \\
\hline
\end{tabular}

Tri, trivalent; Quad, quadrivalent; pdm, pandemic.

no. 4-2018-1188) and all procedures were carried out in accordance with the relevant guidelines and regulations.

\section{RESULTS}

The specific IgE levels against whole vaccines are described in the Figure. Serum samples from 3 age-matched controls (age, $4.0 \pm 0.4$ years; total serum IgE level, $23.9 \pm 5.1 \mathrm{kUA} / \mathrm{L}$ ) were compared. The patient showed elevated specific IgE levels against all of tested whole vaccines rather than controls, especially to vaccines with split virion from egg-based manufacturing process (vaccines $\mathrm{A}-\mathrm{C}$ ). The IgE level to subunit virion vaccine produced in embryonated eggs (vaccine $\mathrm{D}$ ) was lower than that to split virion vaccines, but the IgE response to vaccine $\mathrm{E}$ with subunit virion through cell culture-based manufacturing process was the lowest (Figure A). The specific IgE level to all the tested influenza vaccines decreased over time, but the patient still
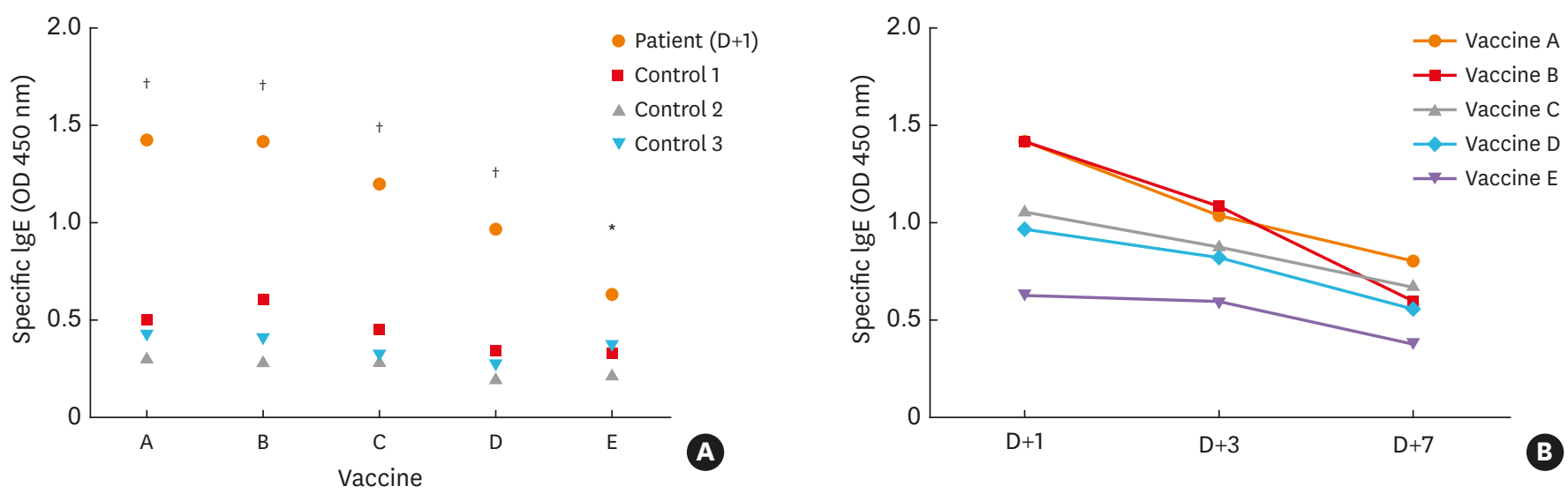

Figure. Measurements of specific IgE levels to influenza vaccines. (A) Comparison of the specific IgE levels against influenza vaccines between a patient and the controls. (B) Changes in the specific IgE levels to influenza vaccines in a patient on days 1, 3, 7 after anaphylaxis.

IgE, immunoglobulin E; OD, optical density.

${ }^{*} P<0.01$ vs. controls, ${ }^{\dagger} P<0.001$ vs. controls. 
showed higher IgE responses at 7 days after anaphylaxis than controls. Only the specific IgE level to vaccine $\mathrm{E}$ measured at 7 days after anaphylaxis event in a patient was as low as that of the controls (Figure B). These results suggested that the patient showed increased IgE responses to influenza vaccines with split virion produced in embryonated eggs rather than with subunit influenza virus vaccine produced in the cell culture systems.

\section{DISCUSSION}

Vaccines contain numerous components that cause allergic reactions. ${ }^{2,5}$ In relation to influenza vaccines, vaccine-associated anaphylaxis has been a concern in egg allergy patients because of classic vaccine production process using embryonated chicken eggs. Egg-based production could increase the chance of egg-adapted changes in viruses. ${ }^{2,7}$ However, the rate of anaphylaxis after influenza vaccination is not greater in recipients with egg allergy than those without. Moreover, egg-free influenza vaccines are known to induce anaphylaxis following vaccination..$^{2,8}$ This might be due to repeated vaccination with influenza hemagglutinin split vaccines, which are reported to induce IgE sensitization against the influenza vaccines, irrespective of the subtypes of influenza viruses. ${ }^{7,9}$ Additionally, in the aspect of viral antigens, split vaccines still contain internal viral proteins despite disruption, which might stimulate CD4 T cells inducing Th2 responses. This might be the reason for undesired allergic responses. ${ }^{7,10}$ However, subunit vaccines containing purified and enriched surface antigens of influenza virus could induce humoral responses alone. ${ }^{79}$ Overall, split formulation of influenza vaccines has been suggested to prevent the potential risk of IgE sensitization or unnecessary immune reactions.

The cases of vaccine-associated anaphylaxis occurring at $>4$ hours after immunization were previously reported, but they were adult patients or children with allergic diseases. ${ }^{2}$ We presented a case of delayed-onset anaphylaxis to influenza vaccination in a child without predisposing allergic diseases. Also, we evaluated IgE responses to influenza vaccines according to the manufacturing process and formation of viral antigens. When we investigated IgE responses to several influenza vaccines from different manufacturers, IgE responses were increased in the patient, especially to an egg-based split influenza vaccine. Moreover, she had a history of repeated immunization with egg-based, trivalent inactivated split influenza virus vaccines in the past and a history of urticaria after vaccination the previous year. We supposed that CD4 T cells stimulated by split viral antigens induced unexpected $\mathrm{Th} 2$ responses, resulting in mast cell activation, IgE production, and finally anaphylaxis. Moreover, based at lowest specific IgE level, cell-based and subunit influenza virus vaccines might be considered safe in this case.

In this case, the symptoms were found 12 hours after exposure and anaphylaxis was diagnosed 24 hours after immunization. It is difficult to explain the exact mechanism or a specific responsible component of this anaphylactic event. Because of the severity of this event and parental disapproval, reimmunization ${ }^{1}$ were not conducted. A very small number of case and no attempt to reimmunization could be limitations to this study. However, based on the ELISA results, we suggest that increased IgE response to influenza vaccine could induce delayed-onset anaphylaxis.

In conclusion, delayed-onset of anaphylaxis may occur following immunization in pediatric patients without predisposing allergic diseases. In addition, the formulation and production system of influenza vaccines could affect the probability of severe allergic reactions to vaccines. 


\section{ACKNOWLEDGMENTS}

The authors thank Thermo Fisher Scientific Korea (Seoul, Korea) for gifting of the immunoCAP liquid reagents ${ }^{\circledR}$.

This work was supported by a grant from the Korea Health Technology R\&D Project through the Korea Health Industry Development Institute (KHIDI) funded by the Ministry of Health \& Welfare, Republic of Korea (grant number HI16C0976, HI15C2971, and HI18C0590); and by the Korea Research Foundation Grant funded by the Korean Government (NRF2015R1D1A1A01061217).

\section{REFERENCES}

1. Dreskin SC, Halsey NA, Kelso JM, Wood RA, Hummell DS, Edwards KM, et al. International Consensus (ICON): allergic reactions to vaccines. World Allergy Organ J 2016;9:32.

PUBMED | CROSSREF

2. McNeil MM, DeStefano F. Vaccine-associated hypersensitivity. J Allergy Clin Immunol 2018;141:463-72. PUBMED | CROSSREF

3. Zafack JG, De Serres G, Rouleau I, Gariépy MC, Gagnon R, Drolet JP, et al. Clinical approach used in medical consultations for allergic-like events following immunization: case series report in relation to practice guidelines. J Allergy Clin Immunol Pract 2017;5:718-727.e1. PUBMED | CROSSREF

4. Nagao M, Fujisawa T, Ihara T, Kino Y. Highly increased levels of IgE antibodies to vaccine components in children with influenza vaccine-associated anaphylaxis. J Allergy Clin Immunol 2016;137:861-7. PUBMED | CROSSREF

5. McNeil MM, Weintraub ES, Duffy J, Sukumaran L, Jacobsen SJ, Klein NP, et al. Risk of anaphylaxis after vaccination in children and adults. J Allergy Clin Immunol 2016;137:868-78. PUBMED | CROSSREF

6. Atanaskovic-Markovic M, Gomes E, Cernadas JR, du Toit G, Kidon M, Kuyucu S, et al. Diagnosis and management of drug-induced anaphylaxis in children: an EAACI position paper. Pediatr Allergy Immunol 2019;30:269-76. PUBMED | CROSSREF

7. Soema PC, Kompier R, Amorij JP, Kersten GF. Current and next generation influenza vaccines: formulation and production strategies. Eur J Pharm Biopharm 2015;94:251-63. PUBMED | CROSSREF

8. Woo EJ. Allergic reactions after egg-free recombinant influenza vaccine: reports to the US Vaccine Adverse Event Reporting System. Clin Infect Dis 2015;60:777-80. PUBMED | CROSSREF

9. Krammer F, Palese P. Advances in the development of influenza virus vaccines. Nat Rev Drug Discov 2015;14:167-82. PUBMED | CROSSREF

10. Nakayama T, Kumagai T, Nishimura N, Ozaki T, Okafuji T, Suzuki E, et al. Seasonal split influenza vaccine induced IgE sensitization against influenza vaccine. Vaccine 2015;33:6099-105.

PUBMED | CROSSREF 